\title{
Response to and toxicity of weekly paclitaxel and carboplatin in patients with stage IIIC-IV ovarian cancer and poor general condition
}

\author{
SUZUYO TAKAHASHI, YUJI TAKEI, KOHEI TAMURA, AKIYO TANEICHI, \\ YOSHIFUMI TAKAHASHI, TAKAHIRO YOSHIBA, TAKAHIRO KOYANAGI, \\ RISA NARUMI, YASUSHI SAGA and HIROYUKI FUJIWARA
}

Department of Obstetrics and Gynecology, Jichi Medical University, Shimotsuke, Tochigi 329-0498, Japan

Received August 15, 2021; Accepted October 22, 2021

DOI: $10.3892 / \mathrm{mco} .2021 .2449$

\begin{abstract}
It has remained elusive whether standard chemotherapy regimens are safe for patients with ovarian cancer and poor general condition. The purpose of the present study was to assess the response to and toxicity of weekly paclitaxel and carboplatin (W-PC) in patients with ovarian cancer and poor general condition. The subjects were patients with ovarian cancer who received W-PC at Jichi Medical University Hospital (Shimotsuke, Japan) between January 2008 and December 2016. Patients who were $\geq 80$ years old and/or had a performance status $\geq 3$ and/or severe complications/underlying diseases were selected. Patients received paclitaxel $\left(60 \mathrm{mg} / \mathrm{m}^{2}\right)$ and carboplatin (area under the curve $2 \mathrm{mg} / \mathrm{ml} / \mathrm{min}$ ) on days 1,8 , and 15 of a 28 -day cycle. Their medical records were retrospectively reviewed. A total of 31 patients were included in the study. Grade 3/4 neutropenia, anemia and thrombocytopenia developed in $18(58 \%), 5(16 \%)$ and $1(3 \%)$ patients, respectively. Furthermore, three (10\%) patients had a complete response (CR), 12 (39\%) had a partial response (PR), $5(16 \%)$ had stable disease and $11(35 \%)$ had progressive disease. The overall response rate was $48 \%(15 / 31)$ and the disease control rate was $65 \%$ (20/31). The 5-year progression-free survival was $15 \%$ and the 5-year overall survival was $15 \%$. A total of 9 patients survived for $>40$ months, one of whom survived without recurrence for 122 months. Performance status $<3$, a tumor response of CR or PR and $>5$ chemotherapy cycles were indicators of favorable prognosis. Only $>5$ chemotherapy cycles (vs. $\leq 5 ; \mathrm{P}=0.002$ ) was an independent good prognostic factor according to multivariate analysis. In conclusion, W-PC was tolerable and slightly effective in patients with ovarian
\end{abstract}

Correspondence to: Dr Yuji Takei, Department of Obstetrics and Gynecology, Jichi Medical University, 3311-1 Yakushiji, Shimotsuke, Tochigi 329-0498, Japan

E-mail: ytakei@jichi.ac.jp

Key words: ovarian cancer, poor general condition, response, toxicity, weekly paclitaxel and carboplatin cancer and poor general condition. W-PC may be one option for patients who are unable to receive standard chemotherapy regimens.

\section{Introduction}

At present, there are 3 major chemotherapeutic regimens for epithelial ovarian cancer: Paclitaxel plus carboplatin every 3 weeks (TW-PC) (1-3), paclitaxel plus carboplatin with bevacizumab followed by bevacizumab maintenance therapy every 3 weeks $(\mathrm{PC}+\mathrm{BEV})(4,5)$ and dose-dense paclitaxel $(80 \mathrm{mg} / \mathrm{m} 2)$ administered on days 1,8 and 15 plus carboplatin [area under the curve (AUC) $6 \mathrm{mg} / \mathrm{ml} / \mathrm{min}$ ] administered on day 1 of a 21-day cycle (dd-PC) (6). Recently, a number of therapeutic regimens using a poly(adenosine diphosphate-ribose) polymerase (PARP) inhibitor were also reported (7-9). One of the above regimens is selected, and whether a PARP inhibitor is used is decided according to the BRCA mutation status and homologous recombination status.

However, it remains elusive whether the above standard regimens may be administered to patients with poor performance status (PS), elderly patients or those with severe complications/underlying diseases. In addition, in the case of patients who receive best supportive care (BSC) due to poor general condition, it remains to be elucidated whether chemotherapy should be administered.

In the present study, a regimen was used including paclitaxel $\left(60 \mathrm{mg} / \mathrm{m}^{2}\right)$ plus carboplatin (AUC $2 \mathrm{mg} / \mathrm{ml} / \mathrm{min}$ ) every week (W-PC) for patients who may be judged as being unable to receive a standard regimen due to poor general condition, i.e., those who may select BSC. The purpose of the present study was to retrospectively assess the response to and toxicity of the W-PC regimen in patients with ovarian cancer and poor general condition.

\section{Materials and methods}

Patients. The medical records of patients who were treated using W-PC for epithelial ovarian cancer, tubal cancer or primary peritoneal cancer at Jichi Medical University 
Hospital (Shimotsuke, Japan) between January 2008 and December 2016 were reviewed. Patients who were $\geq 80$ years old and/or had a PS $\geq 3$ and/or severe complications/underlying diseases were selected. Not only patients who were $\geq 80$ years old but also patients younger than 80 years with PS $\geq 3$ and/or severe complications/underlying diseases were selected. Severe complications and underlying diseases included ileus, pulmonary embolism and pulmonary hypertension. All patients received W-PC as the first-line chemotherapy regimen. Patients who received W-PC as neoadjuvant chemotherapy (NAC) and those who did not receive interval debulking surgery (IDS) after NAC due to poor general condition or chemotherapy resistance were included. All patients had evaluable lesions. Patients who had active double cancer were excluded. W-PC was administered to the above patients with poor general condition. As it was difficult to determine whether the patients were able to receive chemotherapy, W-PC was administered after sufficient informed consent from the patients and their families. The following data were obtained from the medical records: Age, complications, underlying diseases, Eastern Cooperative Oncology Group PS (10), International Federation of Gynecology and Obstetrics (FIGO) stage (11), operative procedure, timing of chemotherapy administration, toxicities, tumor response, progression-free survival (PFS) and overall survival (OS).

The present study was approved by the institutional review board of Jichi Medical University (Shimotsuke, Japan).

Treatment plan. Patients received paclitaxel $\left(60 \mathrm{mg} / \mathrm{m}^{2}\right)$ and carboplatin (AUC $2 \mathrm{mg} / \mathrm{ml} / \mathrm{min}$ ) intravenously on days 1,8 and 15 of a 28 -day cycle. Although a total of 6-9 cycles of W-PC was planned prior to and after surgery, treatments were discontinued when either the disease progressed, unacceptable toxicity developed or the patient refused treatment. If W-PC was effective but cancer lesions remained, up to 12 cycles were allowed. Bevacizumab was not used because the patients in the present study had poor general condition. Granulocyte colony-stimulating factor (G-CSF) was used only if patients had grade 4 neutropenia or grade 3 febrile neutropenia.

Safety assessment. Laboratory tests were performed on each treatment day. Both hematological and non-hematological toxicities were assessed through review of medical records. Toxicities were assessed using the Common Terminology Criteria for Adverse Events v4.0 (12).

Response and survival assessment. In principle, response to treatment was evaluated by computed tomography (CT). When the lesions did not appear on CT but were clearly observed on pelvic examination, this was also used for evaluating the response. The tumor response was assessed based on the Response Evaluation Criteria in Solid Tumors version 1.1 (13). OS was defined as the time that elapsed between the start of treatment and the date of death or last follow-up. PFS was defined as the time that elapsed between the start of treatment and the date of progression or last follow-up. The day of the start of treatment was defined as the operative day in primary debulking surgery (PDS) cases and as the first administration day of W-PC in NAC cases.
Table I. Characteristics of the patients $(n=31)$.

\begin{tabular}{lc}
\hline Characteristic & Value \\
\hline Age, years & $73(30-82)$ \\
Stage & \\
IIIC & $13(42)$ \\
IVA & $6(19)$ \\
IVB & $12(39)$ \\
Performance status & \\
1 & $1(3)$ \\
2 & $9(29)$ \\
3 & $21(68)$ \\
Operation & \\
Performed & \\
Primary debulking & $12(39)$ \\
surgery & \\
Interval debulking & $9(29)$ \\
surgery & \\
None & $10(32)$ \\
Chemotherapy & \\
Neoadjuvant & $19(61)$ \\
Adjuvant & $12(39)$ \\
Complications & \\
DVT, PE & $7(23)$ \\
Ileus & $6(19)$ \\
Underlying diseases & $11(35)$ \\
Hypertension & $5(16)$ \\
Diabetes mellitus & $2(6)$ \\
Dementia & $2(6)$ \\
Renal dysfunction & $1(3)$ \\
Number of chemotherapy & $5(1-12)$ \\
cycles & \\
Number of chemotherapy & \\
doses & \\
\hline Nas & \\
\hline
\end{tabular}

Values are expressed as the median (range) or $\mathrm{n}(\%)$. DVT, deep venous thrombosis; PE, pulmonary embolism; $\mathrm{PH}$, pulmonary hypertension.

Statistical analysis. Statistical analyses were performed using EZR version 1.54 (Saitama Medical Center, Jichi Medical University) (14), which is a graphical user interface for R (The R Foundation for Statistical Computing). More precisely, it is a modified version of $\mathrm{R}$ commander designed to add statistical functions frequently used in biostatistics. The Kaplan-Meier curves were drawn to evaluate PFS and OS. The significance of the difference in survival distribution between subgroups was evaluated using the log-rank test. Variables with $\mathrm{P}<0.05$ in the log-rank test were subsequently entered into the multivariate analysis. Multivariate analysis was performed using Cox's proportional hazards model. $\mathrm{P}<0.05$ was considered to indicate statistical significance. 
Table II. Hematological toxicities by grade.

\begin{tabular}{|c|c|c|c|c|c|}
\hline Toxicity & G1 & $\mathrm{G} 2$ & G3 & G4 & $\mathrm{G} 3+\mathrm{G} 4$ \\
\hline Leucopenia & $3(10)$ & $10(32)$ & $11(35)$ & $2(6)$ & $13(42)$ \\
\hline Neutropenia & $0(0)$ & $8(25)$ & $13(42)$ & $5(16)$ & $18(58)$ \\
\hline Anemia & $0(0)$ & $21(68)$ & $5(16)$ & $0(0)$ & $5(16)$ \\
\hline Thrombocytopenia & $0(0)$ & $2(6)$ & $1(3)$ & $0(0)$ & $1(3)$ \\
\hline
\end{tabular}

Values are expressed as $\mathrm{n}(\%) . \mathrm{G}$, grade.

Table III. Non-hematological toxicities by grade.

\begin{tabular}{|c|c|c|c|c|c|}
\hline Toxicity & G1 & G2 & G3 & G4 & $\mathrm{G} 2+\mathrm{G} 3+\mathrm{G} 4$ \\
\hline AST/ALT & $0(0)$ & $1(3)$ & $1(3)$ & $0(0)$ & $2(6)$ \\
\hline Creatinine & $0(0)$ & $5(16)$ & $2(6)$ & $0(0)$ & $7(23)$ \\
\hline Neuropathy & $7(23)$ & $1(3)$ & $0(0)$ & $0(0)$ & $1(3)$ \\
\hline Nausea & $12(39)$ & $4(13)$ & $0(0)$ & - & $4(13)$ \\
\hline Diarrhea & $3(10)$ & $2(6)$ & $1(3)$ & $0(0)$ & $3(10)$ \\
\hline Alopecia $^{\mathrm{a}}$ & $7(47)$ & 7 (47) & - & - & $7(47)$ \\
\hline
\end{tabular}

${ }^{\mathrm{a} A l o p e c i a ~ w a s ~ e v a l u a b l e ~ f o r ~} 15$ patients based on medical records. Values are expressed as n (\%). G, grade; AST, alanine aminotransferase; $\mathrm{ALT}$, aspartate aminotransferase.

\section{Results}

Patient characteristics. The patient characteristics are summarized in Table I. A total of 31 patients were included in this study. The median age was 73 (range, 30-82) years. The numbers of patients who had FIGO stage IIIC, IVA and IVB were $13(42 \%), 6(19 \%)$ and $12(39 \%)$, respectively. Furthermore, one (3\%), $9(29 \%)$ and $21(68 \%)$ patients had a PS of 1, 2 and 3, respectively. A total of 21 patients $(68 \%)$ underwent surgery. Among them, 12 underwent PDS and 9 underwent IDS. Of all patients, 19 (61\%) received NAC and 12 (39\%) received adjuvant chemotherapy; 10 of the 19 patients who received NAC did not undergo IDS due to poor general condition or chemotherapy resistance. The major complications and underlying diseases were as follows: Deep venous thrombosis and/or pulmonary embolism, 7 (23\%); ileus, 6 (19\%); hypertension, $11(35 \%)$; and diabetes mellitus, 5 (16\%). A total of 11 patients had severe complications and underlying diseases, which were defined as ileus, pulmonary embolism and pulmonary hypertension. The median number of chemotherapy cycles was 5 (range, 1-12) and the median number of chemotherapy doses was 11 (range, 2-30). In the NAC cases, these were the numbers after adding up pre- and post-operative values.

Toxicity. Hematological toxicities are presented in Table II. Grade 3/4 leucopenia, neutropenia, anemia and thrombocytopenia developed in 13 (42\%), 18 (58\%), 5 (16\%), and $1(3 \%)$ patient(s), respectively. G-CSF was used in 4 patients.

Non-hematological toxicities are indicated in Table III. Grade 2 or higher liver dysfunction, renal dysfunction,
Table IV. Tumor response in the cohort.

\begin{tabular}{lc}
\hline Tumor response & Value \\
\hline Complete response & $3(10)$ \\
Partial response & $12(39)$ \\
Stable disease & $5(16)$ \\
Progressive disease & $11(35)$ \\
Response rate, $\%$ & 48 \\
Disease control rate, $\%$ & 65 \\
\hline
\end{tabular}

${ }^{a}$ The 2 patients who discontinued chemotherapy of their own volition after one cycle were included. Values are expressed as n (\%) unless otherwise indicated.

neuropathy, nausea and diarrhea developed in $2(6 \%)$, $7(23 \%), 1(3 \%), 4(13 \%)$ and $3(10 \%)$ of patients, respectively. Grade 2 or higher alopecia, which was possible to evaluate in only 15 patients based on their medical records, developed in 7 (47\%) patients. There were no allergic reactions. A total of 163 cycles were performed, equaling a total of 489 (163 x 3) dose chances, as W-PC involved 3 doses per 1 cycle in principle. Of the 489 dose chances, $392(80 \%)$ doses were administered without skipping. Skipping meant that a dose chance was omitted due to toxicity.

Response and survival. Tumor responses are presented in Table IV. A total of $3(10 \%)$ patients had a complete 
Table V. Univariate analyses of prognostic factors.

\begin{tabular}{|c|c|c|c|}
\hline Variable & Number of patients & Median OS (months) & P-value \\
\hline Age, years & & & 0.874 \\
\hline$<73$ & 15 & 10 & \\
\hline$\geq 73$ & 16 & 30 & \\
\hline Stage & & & 0.705 \\
\hline III & 13 & 7 & \\
\hline IV & 18 & 19 & \\
\hline PS & & & 0.020 \\
\hline 1,2 & 10 & 49 & \\
\hline 3 & 21 & 13 & \\
\hline Chemotherapy & & & 0.813 \\
\hline Neoadjuvant & 19 & 30 & \\
\hline Adjuvant & 12 & 12 & \\
\hline $\begin{array}{l}\text { Complications/underlying } \\
\text { diseases }\end{array}$ & & & 0.126 \\
\hline Non-severe & 20 & 34 & \\
\hline Severe & 11 & 6 & \\
\hline Neutropenia, grade & & & 0.775 \\
\hline$<3$ & 13 & 11 & \\
\hline$\geq 3$ & 18 & 19 & \\
\hline Tumor response & & & $<0.001$ \\
\hline $\mathrm{CR} / \mathrm{PR}$ & 15 & 42 & \\
\hline $\mathrm{SD} / \mathrm{PD}$ & 16 & 6 & \\
\hline $\begin{array}{l}\text { Number of chemotherapy } \\
\text { cycles }\end{array}$ & & & $<0.001$ \\
\hline$>5$ & 15 & 49 & \\
\hline$\leq 5$ & 16 & 6 & \\
\hline
\end{tabular}

CR, complete response; OS, overall survival; PD, progressive disease; PR, partial response; PS, performance status; $\mathrm{SD}$, stable disease.

Table VI. Multivariate analysis of prognostic factors.

\begin{tabular}{lccc}
\hline Variable & Number of patients & Hazard ratio & 95\% CI \\
\hline PS & 10 & 1 & $0.85-6.98$ \\
1,2 & 21 & 2.43 & 0.098 \\
3 & & & $0.89-8.03$ \\
Tumor response & 15 & 1 & 0.080 \\
CR/PR & 16 & 2.67 & $2.26-36.6$ \\
SD/PD & & & 0.002 \\
Number of & 15 & 1 & \\
chemotherapy cycles & 16 & 9.09 & \\
$>5$ & 16 & & \\
$\leq 5$ & & &
\end{tabular}

95\% CI, 95\% confidence interval; CR, complete response; PD, progressive disease; PR, partial response; PS, performance status; SD, stable disease.

response (CR), $12(39 \%)$ had a partial response (PR), $5(16 \%)$ had stable disease and $11(35 \%)$ had progressive disease (PD). Patients with PD included 2 who discontinued chemotherapy of their own volition after one cycle. The overall response rate was $48 \%(15 / 31)$ and the disease control rate was $65 \%(20 / 31)$. The median follow-up period was 
A

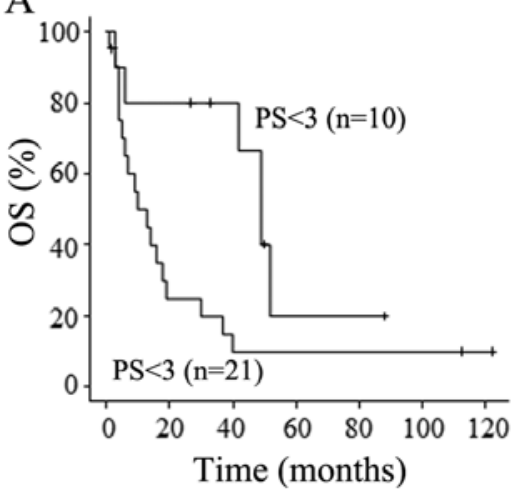

B

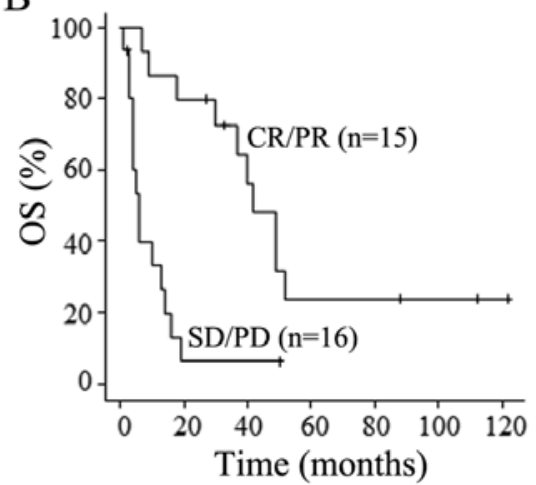

C

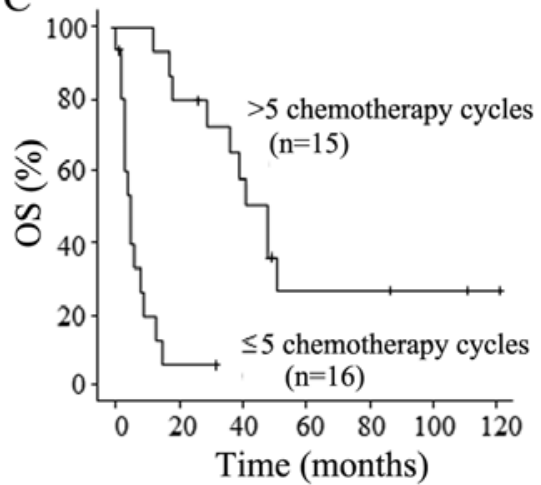

Figure 1. Kaplan-Meier curves. (A) OS curves according to PS. (B) OS curves according to the response to chemotherapy. (C) OS curves according to the number of chemotherapy cycles. A total of two patients were censored at 2 and 50 months, respectively. OS, overall survival; PS, performance status; CR, complete response; PR, partial response; $\mathrm{SD}$, stable disease; PD, progressive disease.

16 (1-122) months. The median PFS period was 12 months and the 5-year PFS rate was $15 \%$. The median OS period was 18 months and the 5-year OS rate was $15 \%$. A total of 9 patients survived for $>40$ months, 3 of whom had no recurrence. The patient who survived for the longest period survived with no recurrence for 122 months.

The results of the univariate analyses of prognostic factors are provided in Table $\mathrm{V}$. $\mathrm{PS}<3$, tumor response of $\mathrm{CR}$ or PR and $>5$ chemotherapy cycles were favorable prognostic factors. Only $>5$ chemotherapy cycles was an independent factor for favorable prognosis according to multivariate analysis (Table VI). However, age, stage, timing of chemotherapy administration, severe complications/underlying diseases and toxicities did not have any prognostic value. OS curves according to PS, response to chemotherapy and number of chemotherapy cycles are presented in Fig. 1.

\section{Discussion}

In the present study, W-PC was selected for patients with ovarian cancer who were unable to receive a standard chemotherapy regimen due to having poor general condition. The efficacy and safety of W-PC for patients with ovarian cancer and poor general condition was retrospectively examined. The results demonstrated that $\mathrm{W}-\mathrm{PC}$ exhibited tolerable toxicity and was slightly effective in patients with ovarian cancer and poor general condition.

W-PC had tolerable toxicity in patients with ovarian cancer who were unable to receive a standard regimen such as TW-PC, PC-BEV or dd-PC due to having poor general condition. These standard regimens are likely to be markedly toxic to patients with poor general condition, preventing them from continuing chemotherapy or worsening their general condition. In the TW-PC group of the Multicenter Italian Trials in Ovarian cancer (MITO) 7 trial (15) and Japanese Gynecologic Oncology Group (JGOG) 3016 trial (6), grade 3-4 neutropenia rates were 50 and $88 \%$ and grade 3-4 thrombocytopenia rates were 7 and 38\%, respectively. The patients in the JGOG 3016 trial exhibited more severe myelosuppression than those in the MITO 7 trial, suggesting that the Japanese patients exhibited slightly more severe myelosuppression. The reason may be polymorphisms in genes that function in taxane metabolism (16). Therefore, it is difficult to administer TW-PC safely to Japanese patients with poor general condition. However, W-PC may be safe for Japanese patients with poor general condition. In the MITO 7 trial, patients assigned to $\mathrm{W}-\mathrm{PC}$ had milder toxicities than patients assigned to TW-PC (15).

In the JGOG 3016 trial, the dd-PC group had more severe anemia than the TW-PC group (6). This may have been because the paclitaxel dose per week in the dd-PC group was higher than that in the TW-PC group ( $80 \mathrm{vs.} 60 \mathrm{mg} / \mathrm{m}^{2}$, respectively). The paclitaxel dose per administration in the W-PC regimen of the present study was $60 \mathrm{mg} / \mathrm{m}^{2}$, which may be safe for patients with poor general condition. Since the patients of the present study had a poor general condition and were not able to receive chemotherapy itself, a lower dose (paclitaxel $60 \mathrm{mg} / \mathrm{m}^{2}$ ) was selected for safety. W-PC in the MITO 7 trial was administered on days 1, 8 and 15 of a 21-day cycle. However, in the present study, the W-PC regimen was administered on days 1,8 and 15 of a 28-day cycle. A 28-day cycle regimen was considered to be safer than a 21-day cycle regimen for patients with poor general condition. Watanabe et al (17) used the same W-PC regimen as in the present study; their regimen was also administered in a 28-day cycle. Their patients had platinum-sensitive relapsed ovarian cancer and a PS of 0-2, which was better than the condition of the patients of the present study. They reported a rate of grade 3-4 neutropenia of 32\% and a rate of grade 3-4 thrombocytopenia of $0 \%$ (17). Grade 3-4 neutropenia was observed more frequently in the present study (58\%), as the present cases had a poorer general condition. Therefore, the present regimen in a 28-day cycle for patients with poor general condition may be appropriate. If a 21-day cycle had been adopted, the toxicities may have been more severe.

In the present study, W-PC was slightly effective in patients with ovarian cancer and poor general condition. A similar study recently reported that NAC with W-PC for patients with ovarian cancer and poor PS reduced the toxicity of chemotherapy and had the same efficacy as TW-PC (18). Their W-PC regimen was also administered in a 28-day cycle. Response and disease control rates in their W-PC group were $70 \%(14 / 20)$ and $80 \%(16 / 20)$, respectively, being higher than those in the present study. One reason may be that the 
patients in the present study had a poorer general condition. For instance, the median age of patients in the present study and their study was 73 (30-82) and 61 (36-75), respectively, and the rate of patients with a PS of 3 was 68 and $40 \%$, respectively. The ICON8 trial compared 3 regimens of TW-PC, W-PC and dd-PC. The PFS of the three groups was similar (24.4, 25.3 and 24.9 months, respectively) (19). The W-PC regimen in the ICON8 trial was paclitaxel $(80 \mathrm{mg} / \mathrm{m} 2)$ and carboplatin (AUC $2 \mathrm{mg} / \mathrm{ml} / \mathrm{min}$ ) administered on days 1,8 and 15 of a 21-day cycle, whereas the W-PC regimen of the present study was paclitaxel $\left(60 \mathrm{mg} / \mathrm{m}^{2}\right)$ and carboplatin (AUC $2 \mathrm{mg} / \mathrm{ml} / \mathrm{min}$ ) administered on days 1,8 and 15 of a 28-day cycle. Although the W-PC regimen in the ICON8 trial differed from the W-PC regimen of the present study, W-PC may have similar efficacy to TW-PC and dd-PC. However, sufficient evidence as to whether the present W-PC regimen may be applied to patients with ovarian cancer and good general condition is lacking.

In the present study, the W-PC regimen was selected for patients who may receive $\mathrm{BSC}$ due to advanced age, $\mathrm{PS} \geq 3$ or severe complications/underlying diseases. Of these patients with poor general condition, 9 survived for 40 months or longer and 3 had no recurrence. Furthermore, one patient survived with no recurrence for 122 months. According to multivariate analysis, $>5$ chemotherapy cycles was the only independent favorable prognostic factor. The 9 patients who survived for $>40$ months all received $>5$ cycles of W-PC. The number of chemotherapy cycles may be a valuable prognostic factor that reflects numerous elements such as tumor response and adverse effects. In the W-PC regimen, unlike in TW-PC and dd-PC, both paclitaxel and carboplatin are administered in divided doses from day 1 . In patients with poor general condition, severe adverse effects may develop or general conditions may deteriorate after the administration of anticancer agents. If an anticancer agent is administered in a small dose on day 1 , such as in W-PC, the development of severe adverse effects and the deterioration of the general condition may be minimized. Therefore, W-PC is safe for patients with poor general condition. These methods are also performed for other types of carcinoma, such as urothelial carcinoma (20).

The current study had certain limitations due to its retrospective design. First, sufficient data on adverse events other than blood test results were unavailable, but the results may not be significantly altered as severe toxicity was confirmed. The reason is as follows: Since almost all severe toxicities of neuropathy, nausea, diarrhea and alopecia have apparent symptoms, it was unlikely that any toxicities remained unnoticed. Furthermore, it was planned to compare the present $\mathrm{W}-\mathrm{PC}$ with the standard regimens, such as TW-PC, PC-BEV and dd-PC; however, the subjects of the present study were ineligible for standard TW-PC, PC-BEV or dd-PC. At our hospital, these 3 regimens are not used for patients with poor general condition, precluding comparisons between W-PC and the above regimens.

In conclusion, W-PC exhibited tolerable toxicity and was slightly effective in patients with ovarian cancer with poor general condition. In addition, W-PC may lead to long-term survival, albeit in only a small number of patients. If patients receive $>5$ cycles of $\mathrm{W}-\mathrm{PC}$, their survival may be prolonged.
Thus, W-PC may be an appropriate therapeutic option for patients for whom standard regimens are contraindicated due to advanced age, poor PS or severe complications/underlying diseases.

\section{Acknowledgements}

Not applicable.

\section{Funding}

No funding was received.

\section{Availability of data and materials}

The datasets used and/or analyzed during the current study are available from the corresponding author upon reasonable request.

\section{Authors' contributions}

ST, YT, and HF designed the present study, critically revised the manuscript and analyzed data. KT, AT, YT, TY, TK, RN and YS retrieved the data from the medical records, confirmed the authenticity of the raw data and revised the work critically for important intellectual content. ST and YT wrote the manuscript. All authors read and approved the final manuscript.

\section{Ethics approval and consent to participate}

This study was approved by the ethics committee of Jichi Medical University (Shimotsuke, Japan). The ethics committee of Jichi Medical University (Shimotsuke, Japan) judged that patient consent was not required as this was a retrospective observational study. The patients or their families were given the opportunity to opt out.

\section{Patient consent for publication}

Not applicable.

\section{Competing interests}

The authors declare that they have no competing interests.

\section{References}

1. Ozols RF, Bundy BN, Greer BE, Fowler JM, Clarke-Pearson D Burger RA, Mannel RS, DeGeest K, Hartenbach EM, Baergen R and Gynecologic Oncology Group: Phase III trial of carboplatin and paclitaxel compared with cisplatin and paclitaxel in patients with optimally resected stage III ovarian cancer: A Gynecologic Oncology Group study. J Clin Oncol 21: 3194-3200, 2003.

2. du Bois A, Lück HJ, Meier W, Adams HP, Möbus V, Costa S, Bauknecht T, Richter B, Warm M, Schröder W, et al: A randomized clinical trial of cisplatin/paclitaxel versus carboplatin/paclitaxel as first-line treatment of ovarian cancer. J Natl Cancer Inst 95: 1320-1329, 2003.

3. Takei Y, Suzuki M, Ohwada M, Saga Y, Kohno T, Machida S and Sato I: A feasibility study of paclitaxel and carboplatin therapy in Japanese patients with epithelial ovarian cancer. Oncol Rep 10: 951-955, 2003. 
4. Burger RA, Brady MF, Bookman MA, Fleming GF, Monk BJ, Huang H, Mannel RS, Homesley HD, Fowler J, Greer BE, et al: Incorporation of bevacizumab in the primary treatment of ovarian cancer. N Engl J Med 365: 2473-2483, 2011.

5. Perren TJ, Swart AM, Pfisterer J, Ledermann JA, Pujade-Lauraine E, Kristensen G, Carey MS, Beale P, Cervantes A, Kurzeder C, et al: A phase 3 trial of bevacizumab in ovarian cancer. N Engl J Med 365: 2484-2496, 2011.

6. Katsumata N, Yasuda M, Takahashi F, Isonishi S, Jobo T, Aoki D, Tsuda H, Sugiyama T, Kodama S, Kimura E, et al: Dose-dense paclitaxel once a week in combination with carboplatin every 3 weeks for advanced ovarian cancer: A phase 3, open-label, randomised controlled trial. Lancet 374: 1331-1338, 2009.

7. Moore K, Colombo N, Scambia G, Kim BG, Oaknin A, Friedlander M, Lisyanskaya A, Floquet A, Leary A, Sonke GS, et al: Maintenance olaparib in patients with newly diagnosed advanced ovarian cancer. N Engl J Med 379: 2495-2505, 2018.

8. González-Martín A, Pothuri B, Vergote I, DePont Christensen R, Graybill W, Mirza MR, McCormick C, Lorusso D, Hoskins P, Freyer $\mathrm{G}$, et al: Niraparib in patients with newly diagnosed advanced ovarian cancer. N Engl J Med 381: 2391-2402, 2019.

9. Ray-Coquard I, Pautier P, Pignata S, Pérol D, González-Martín A, Berger R, Fujiwara K, Vergote I, Colombo N, Mäenpää J, et al: Olaparib plus Bevacizumab as First-Line Maintenance in Ovarian Cancer. N Engl J Med 381: 2416-2428, 2019.

10. Cancer Therapy Evaluation Program: Common Toxicity Criteria Version 2.0, 1999. http://ctep.cancer.gov/protocolDevelopment/ electronic_applications/docs/ctcv20_4-30-992.pdf. Accessed October 23, 2021

11. Prat $\mathbf{J}$ and FIGO Committee on Gynecologic Oncology: Staging classification for cancer of the ovary, fallopian tube, and peritoneum. Int J Gynaecol Obstet 124: 1-5, 2014.

12. National Cancer Institute: Common Terminology Criteria for Adverse Events. Version 4.0. https://ctep.cancer.gov/protocoldevelopment/electronic_applications/ctc.htm. Accessed August 15, 2021.
13. Eisenhauer EA, Therasse P, Bogaerts J, Schwartz LH, Sargent D, Ford R, Dancey J, Arbuck S, Gwyther S, Mooney M, et al: New response evaluation criteria in solid tumours: Revised RECIST guideline (version 1.1). Eur J Cancer 45: 228-247, 2009.

14. Kanda Y: Investigation of the freely available easy-to-use software 'EZR' for medical statistics. Bone Marrow Transplant 48: 452-458, 2013.

15. Pignata S, Scambia G, Katsaros D, Gallo C, Pujade-Lauraine E, De Placido S, Bologna A, Weber B, Raspagliesi F, Panici PB, et al: Carboplatin plus paclitaxel once a week versus every 3 weeks in patients with advanced ovarian cancer (MITO-7): A randomised, multicentre, open-label, phase 3 trial. Lancet Oncol 15: 396-405, 2014.

16. Ma BB, Hui EP and Mok TS: Population-based differences in treatment outcome following anticancer drug therapies. Lancet Oncol 11: 75-84, 2010

17. Watanabe $\mathrm{Y}$, Nakai $\mathrm{H}$, Ueda $\mathrm{H}$ and Hoshiai $\mathrm{H}$ : Evaluation of weekly low-dose paclitaxel and carboplatin treatment for patients with platinum-sensitive relapsed ovarian cancer. Gynecol Oncol 96: 323-329, 2005.

18. Takaya H, Nakai H, Murakami K, Tobiume T, Suzuki A, Mandai M and Matsumura N: Efficacy of weekly administration of paclitaxel and carboplatin for advanced ovarian cancer patients with poor performance status. Int J Clin Oncol 23: 698-706, 2018

19. Clamp AR, James EC, McNeish IA, Dean A, Kim JW, O'Donnell DM, Hook J, Coyle C, Blagden S, Brenton JD, et al: Weekly dose-dense chemotherapy in first-line epithelial ovarian, fallopian tube, or primary peritoneal carcinoma treatment (ICON8): Primary progression free survival analysis results from a GCIG phase 3 randomised controlled trial. Lancet 394: 2084-2095, 2019.

20. Han JJ, Kim YJ, Kim JW, Chang H, Lee JO, Lee KW, Jeong CW, Kim JH, Hong SK, Bang SM, et al: Salvage treatment with low-dose weekly paclitaxel in elderly or poor performance status patients with metastatic urothelial carcinoma. Tumori 100 : 439-445, 2014. 